\title{
Intelligent High Resolution Satellite/Aerial Imagery
}

\author{
Nadeem Fareed \\ Department of Meterology, Comsats Institute of Information Technology, Islamabad, Pakistan \\ Email: nadeemfareed32@gmail.com, nadeem qln@yahoo.com
}

Received 13 September 2013; revised 13 October 2013; accepted 20 October 2013

Copyright (C) 2014 by author and Scientific Research Publishing Inc.

This work is licensed under the Creative Commons Attribution International License (CC BY). http://creativecommons.org/licenses/by/4.0/

(c) (i) Open Access

\begin{abstract}
High resolution satellite images are rich source of geospatial information. Nowadays, these images contain finest spectral and spatial information of ground realities in different electromagnetic spectrum. Many image processing softwares, algorithms and techniques are available to extract such information from these images. Multi spectral as well as panchromatic (PAN) high resolution satellite images are missing, one important information, regarding ground features and realities that information is attribute information which is not directly available in high resolution satellite images. From very first day, this information used to be collected through indirect ways using GPS, digitizing, geo-coding, geo tagging, field survey and many other techniques. Our real world has vertical labels for ground observer to identify and use this information. These vertical labels are present in form of names, logos, icons, symbols and numbers. These vertical labels ease us to work in real world. Satellites are unable to read these labels due to their vertical orientation. Making satellite/aerial imagery rich of attribute information, we have the possibility to design our world accordingly. Just like vertical labels we can also place real physical horizontal label for space sensors, to make this information directly available in high resolution satellite/aerial imagery. This work is about possibilities of such techniques and methods.
\end{abstract}

\section{Keywords}

High Resolution Satellite Images; Vertical Labels; Horizontal Labels; Physical Labels; Aerial Imagery; Disaster

\section{Introduction}

Panchromatic (PAN) satellite imagery has been used successfully in different applications such as topographic mapping and terrain modelling. Nevertheless, PAN imagery is still one of the less used digital sources for land-change studies except few processes where the high-resolution of the PAN images is used to improve the visualization quality of the multi-spectral images [1]. Panchromatic (PAN) satellite imagery has one big power- 
ful quality; these images contain very high spatial detail. Spatial resolution of these images is increasing rapidly with time. Horizontal labelling scheme is a new frontier defined as a new destiny of panchromatic band.

\section{Digital Data Collection}

In modern era, every physical ground phenomena is changing so rapidly that traditional and old sources are not enough to manage the present needs. Manmade structures are defining the new horizon, while natural disaster vanishing the old ones. So, such changes cannot be accommodated without help of high resolution satellite images. Nowadays, almost everywhere in the world, people are using high resolution images to collect the digital data. People use high resolution satellite images in form of printed sheets to collect the attribute information of manmade structures. Many web applications are designed for such purpose alike.

Remote sensing and satellite imagery are new things in modern world. A lot of good things have done to utilize these sources to help us to understand the world but, as science advances, it grows complex and more demanding. The science of high resolution satellite imagery is demanding new horizons as well as new directions to work.

\section{Intelligent High Resolution Satellite Imagery}

When I see a high resolution satellite image it make me happy for a while then very next moment its make me sad. We designed such a great sensors and platform to scan the earth sources and map them for us. But as far as our side is concern we did not help them to map the most, map the best.

It seems to me stupid that a very vast region is mapped by satellite just in few minutes and we human at same time collecting the very same information with field survey with huge consumption of time.

We can make satellite images more intelligent by number of ways, let look at our world assuming humans as a little satellites operating in passive mode.

\subsection{Vertical or 90 Degree Labels}

Every human being rural or urban, experience I named "vertical or 90 degree labels" almost every day in their life. We, as I do supposed, little satellites, are intelligent enough to gather information around us because our surroundings are intelligent enough. Every day we experience vertical labels around us in form of street signs, placards, banners and beautiful commercial advertisements (Figures 1-4).

These are such fancies we have in our real world. Could you imagine the life such a boring and frustrating without all this stuff? So, infect we are not intelligent enough but our surrounding is.

Do you think that space satellite have such fancies?

And the answer is no. again question is why not?

\subsection{Horizontal or 0 Degree Labels}

Now, the resolution of satellite images is getting batter rapidly, satellites operators are optimistic about this, one day we will have such a resolution level that a person will be able to distinguish a car from a motorbike in a parking lot. Now, we have sub meter resolution. So, question is did we benefited from such a high resolution so far? The answer is no. but we will be in future if we think logically and practically. Take it serious as problem but not as a science fiction. Take it as a solution to many problems but not as a problem itself. It is perceivable so it is achievable logically.

Once I was part of data collector team for a company, they utilized the high resolution satellite images to collect the vertical labels, most the work, now days, and people are doing the same thing for data collection. Sometime I wonder why we don't have satellite images with labels, to avoid such a laborious work. The state forward answer is, because we have vertical labels and satellites do not have ability to map vertical label not physically but technically. So as a result we are missing this information in high resolution images. Which infect our negligence not the sensors problem?

As we can calibrate the sensors to collect high resolution images, we can also calibrate the manmade structures to make it possible for space sensors to map the attribute information with real geometry of ground objects. Natural phenomena are beyond our approach. Now, question is how it's possible?

A simple answer is. Put the horizontal or zero degree labels too. 


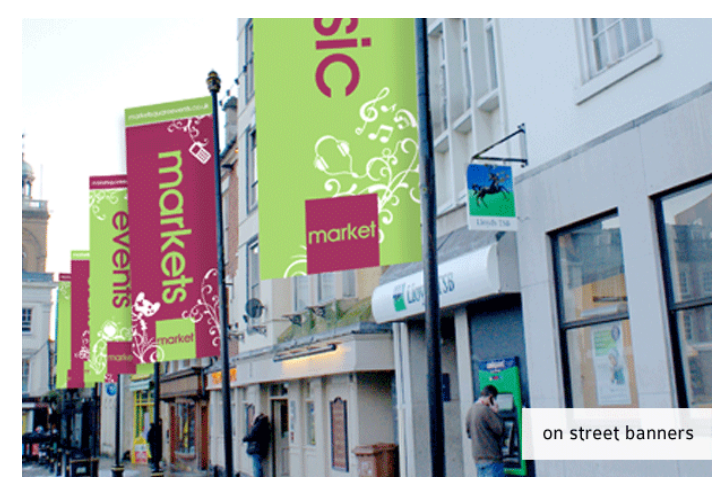

Figure 1. Vertical banners [2].

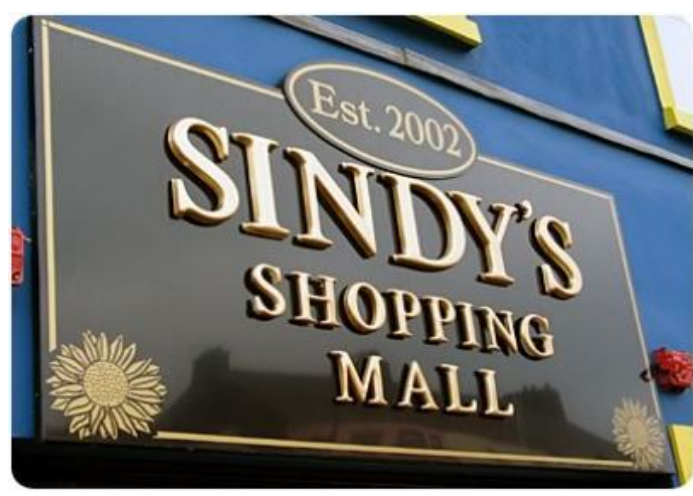

Figure 2. Vertical logo sign [3].

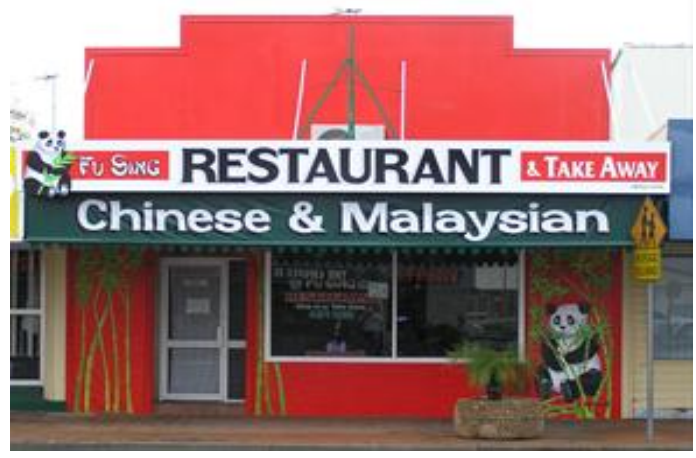

Figure 3. Vertical alphabetical name [4].

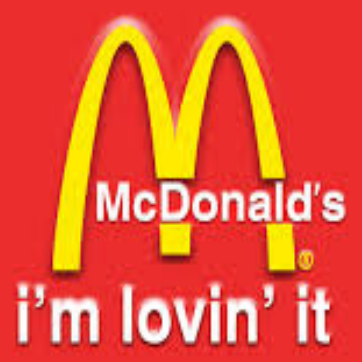

Figure 4. Vertical logo sign [5]. 
Commercial plazas, hotels, motels, shops and residential buildings, all these have one thing common and unoccupied, that is the roof. So, we can place horizontal labels too, for a space sensor to maps us, not only as an object or building, but also map us who we are? And what we do. How long been we are here. As vertical labels are not visible to space sensor, similarly, horizontal labels are not visible to ground observer, this thing, in fact, do not distort the beauty of our real world, but make our world more beautiful and easy to understand spatially.

\section{Methodology}

Every manmade structure either it's a big company, hotel, business place or a residential building. It may have a logo or icon, a name or address in form of alphabets and numbers, or can have a cartographic representation. It's naming fall under one of the following category. So, three categories are defined as under.

- Cartographic signs labeling

- Logos or icons labeling

- Alphabetical and numerical labeling

\subsection{Cartographic Signs Labelling}

Most of the building labels are cartographic signs now days. People who digitize at a GIS platform or web interface they have option for symbols for different features. One big example is OpenStreetMap platform. Cartographic symbols are basic building blocks of a beautiful map, a makeup toolkit for appealing mapping applications. Most of them represent the manmade structures. We leave the natural places as an exceptions, because they carry their own information in satellite images at some extent, the rest of extent is not hard to find out.in some cases as airports, bus stations, train stations, fuel stations, places of worships e.g. mosques, they carry their real cartographic signs and are identifiable in high resolution satellite images. But in most of the cases of manmade structures, it's very hard to define their physical presence in high resolution satellite images. Practically, it is possible to define their physical objective of presence (Figure 5).

These symbols are possible to place physically as real objects on building roofs, backyards, or anywhere at a suitable place directly facing sky.

As far as this research is concern, we want to place these labels as physical labels, we want to make satellite imagery representing not only the real shape and location of a manmade structure but also its attribute information as well. These labels either could be colorful or black and white. In case of black and white labels, the panchromatic (PAN) band has batter spatial resolution so it would be far easier to map these labels with smaller size comparatively. At the same time the spatial resolution of panchromatic (PAN) as well as multispectral bands is increasing each year. So, we have to consider this thing too that how least resolution is possible to achieved in future. This calculation or estimation will help us to design more flexible and accurate system for horizontal labelling.

One best example of such work is visible in geoeye-1 1st high resolution satellite image Kutztown University Pennsylvania. A horizontal label (Figure 6).

\subsection{Logo or Icon Labelling}

Almost every business project either big or small has its own logo or icon. Such companies can also put their icon or logos as horizontal labels too. Same is in given Figure 7. This is an occasional label, but it can be more thoughtful or attractive as given in the Figure 7.

Every business place can be labeled in same way. Logo placed here are images downloaded from internet, such logos can be painted on the roof, black and white version can deliver best for panchromatic (PAN) band of different sensors. Usually panchromatic band have batter resolution as compare to the multispectral bands.

\subsection{Alphabetical and Numerical Labelling}

Residential buildings mostly do not have logos or icons instead they have addresses which contain alphabets and numbers. Individual houses and residential apartments mostly fall under this category. We can paint the house number or numbers on the roof. Most of the addresses are a combination of alphabets and numbers (Figure 8).

This is true that such labeling scheme is a difficult job for residential buildings because every building does not meet the desired labeling orientation but it is not impossible at all. A bucket of black and white paint can do 


\begin{tabular}{|c|c|c|c|}
\hline \multicolumn{4}{|l|}{ UBD Map Symbols } \\
\hline $\begin{array}{l}\text { Airport - Domeatic } \\
\text { Airport - International } \\
\text { Ambulance Station } \\
\text { Barbecue } \\
\text { Boost fyeling point } \\
\text { Bowling club } \\
\text { Bus Stop } \\
\text { Camping Area } \\
\text { Carrvan Park } \\
\text { Par Park }\end{array}$ & 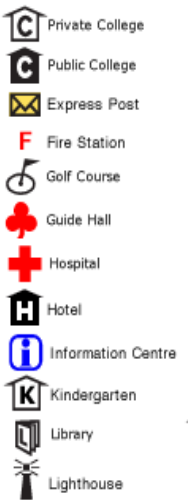 & 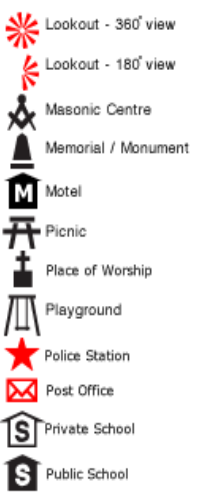 & 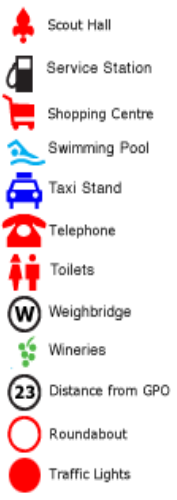 \\
\hline
\end{tabular}

Figure 5. Cartographic symbols of different physical manmade structures [6].

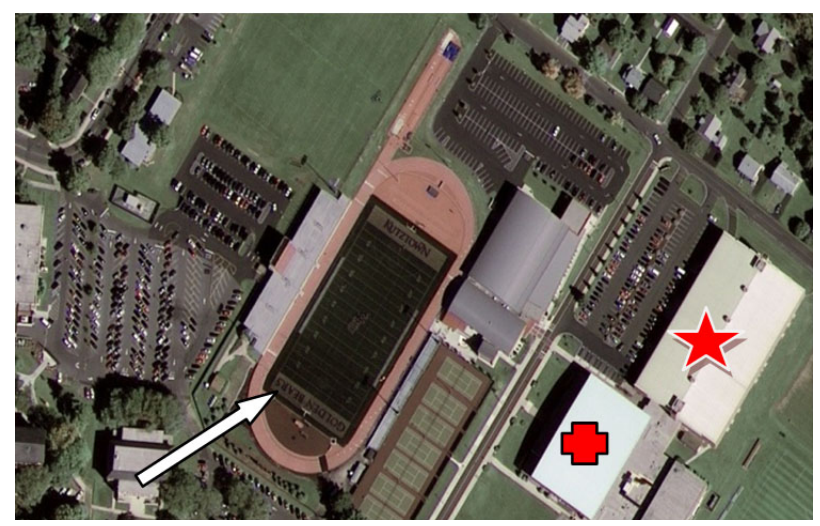

Figure 6. Geoeye-1 1st high resolution image which contain a labeled building. GOLDEN BEARS. Red symbols are supposed cartographic symbols that can be placed over such buildings [7].

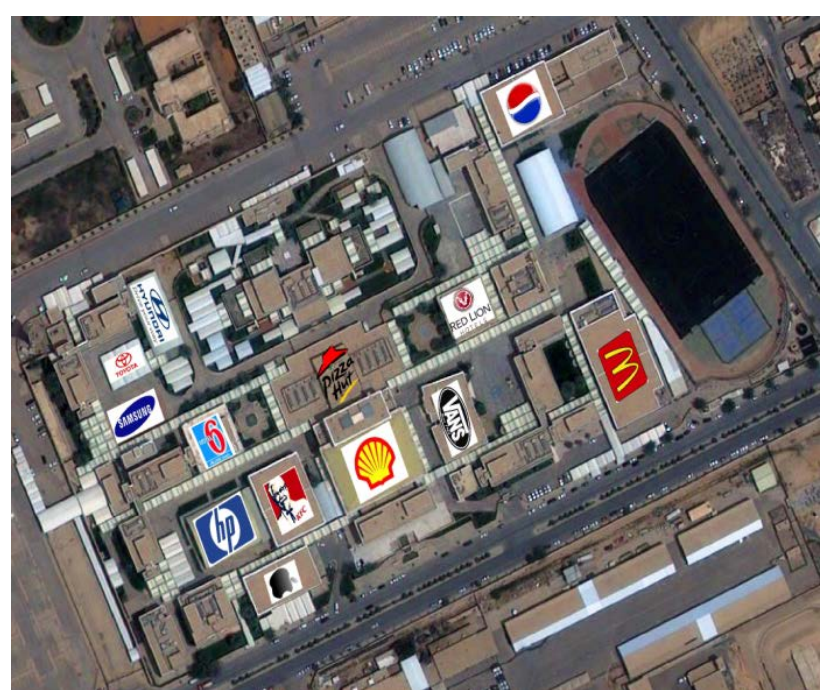

Figure 7. Logo labeling scheme of buildings. The labels demonstrated here are fictions and do not belong to labeled buildings [8]. 
accomplish this job easily.

Figure 9 is an example of well planned and organized area in London UK. such countries have well planned and modernized buildings with complex structures, which makes somehow this labeling scheme difficult. But such countries already have up to date GIS datasets with complete and accurate addresses.

Developing countries have simple and flat roof buildings almost 80 buildings in developing countries have flat roof buildings. Flat roofs are ideal places for putting the alphabetical and numerical labels.

\subsection{Real world Examples}

Here, some real world examples are given below.

\section{Resolution and Visibility}

In the past few years, with the development of imaging techniques, satellites with very high spatial resolution imaging systems have been launched [1]. Spatial resolution has improved in a very short span of time. Further improvement is expected but have some restrictions imposed by the govt. administration.

Following table summarize the spatial resolution of some well known high resolution sensors (Table 1).

All vertical labels small or big are visible to ground observer, because the distance between observer and label is not more than few meters to few hundred meters. But in case of horizontal label, the observer is several hundred of kilometers above the ground level. So, mapping such small labels would not be possible for the sensors if the size

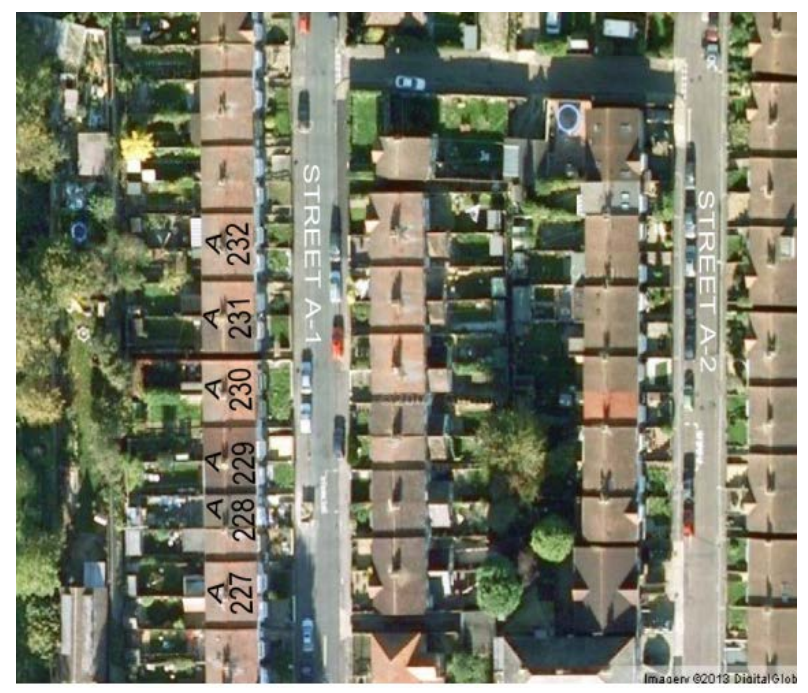

Figure 8. A well planned and organized area in London: Alphabetical and numerical labeling scheme for residential buildings and streets. Streets and roads can also be labeled in same way [9].

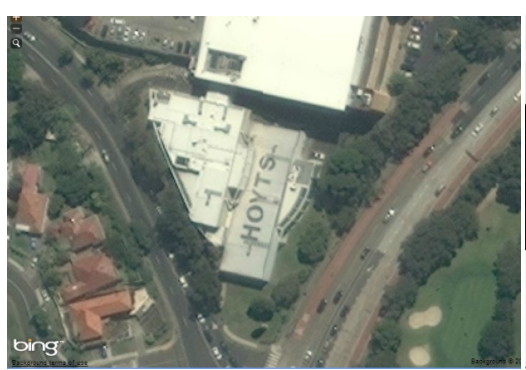

(a)

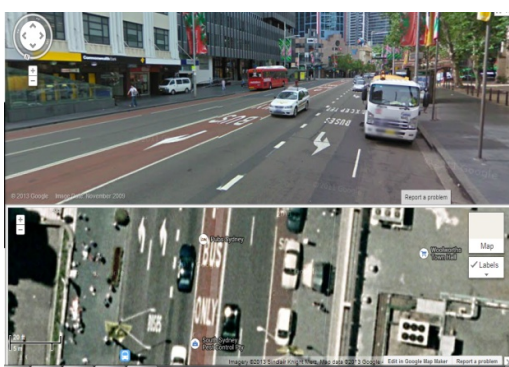

(b)

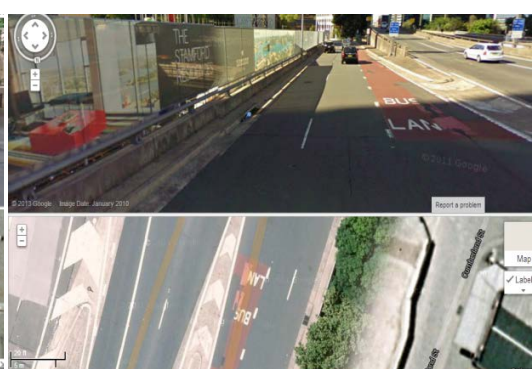

(c)

Figure 9. Real world examples: (a) HOYTS Sydney Australia [10]; (b) Google Earth Sydney Australia [11]; (c) Google Earth Sydney Australia [11]. 
Table 1. Sensor vs. Resolution [12].

\begin{tabular}{cc}
\hline Sensor & Resolution (PAN) \\
WorldView-1 & $0.5 \mathrm{~m}$ \\
Worldview-2 & $0.5 \mathrm{~m}$ \\
Quick bird & $0.6 \mathrm{~m}$ \\
Pleiades 1A/1B & $0.7 \mathrm{~m}$ \\
Geoeye-1 & $0.5 \mathrm{~m}$ \\
EROS & $0.7 \mathrm{~m}$ \\
IKONOS & $0.8 \mathrm{~m}$ \\
\hline
\end{tabular}

or scale of the label remain same. All labels should meet the minimum criteria to map the horizontal labels.

Today, we have the spatial resolution in sub-meter level. So far each label to be visible enough to carry information, its resolution generally should be more than sub-meter level and a more generalize calculation suggest, labels should have minimum one meter resolution to be mapped in panchromatic band. This generalization is an estimation to facilitate the maximum number of sensors to capture the information. This would speed up the mapping rate and global coverage.

Mapping horizontal labels in multispectral bands would be hard thing at 1st instance because a reasonable size is required to map the labels in multispectral bands. Larger size demand leads this project toward fantasy world as compare to a realistic and helping approach. So, this approach is more realistic toward panchromatic band as compare to multispectral band. Similarly, we cannot afford larger size in case of alphabetical and numerical labeling schemes, because this labeling scheme is supposed to cover the residential buildings and residential building do not have much space for large physical labels.

\section{Aerial Photography}

Same problem is the problem with aerial photography. Aerial imagery has finest spatial resolution but missing attributes information. Aerial imagery is not available worldwide but its spatial resolution is amazing as compare to satellite imagery.

Due to this capability of aerial imagery, even small logo or icon are possible to map with great accuracy and visibility.

\section{Labelling Materials and Methods}

One of the best material that generally come into the mind is "two buckets of permanent paint" one with black tone and other with white. A white label with black background would give the best results in panchromatic (PAN) band. This method is best for residential buildings labeling scheme.

Big commercial buildings and well known companies are supposed to adopt the logo or icon labeling schemes because they have both, a good space for label and handsome funding for attractive labeling. Advertising through space is not a bad idea at all.

Govt. buildings and organizations can follow the cartographic labeling scheme which suits them best. The reason behind this supposition is that such buildings have lengthy name and addresses, managing their horizontal labels is a hard thing. But, they can follow the logo or icon labeling scheme too, depending upon space availability and funding for such labels. One thing more, they must have a logo or icon.

This is just an idea, standard can be defined later.

\section{Privacy and Objections}

This project is a success, most of the buildings has label as desired. But many secret agencies and companies would not like to put their physical labels openly to map for anyone globally. This will lead to a privacy threat to many government organizations and agencies. So, simple solution to this problem is fake labels to keep the 
momentum with the rest of system. Fake labelling scheme will make the privacy invincible among the invaders. You have no solution to differentiate between a true label and a fake label without ground visit. So people sitting far side the country would not be able to find the true location as a target.

\section{Advantages and Disadvantages}

Expected advantages and disadvantages are mention here below.

\subsection{Advantages}

Such labeling schemes will enrich the satellite images with attribute information at world scale. The following benefits are sure from such images.

- Many NGOs are using high resolution satellite images to digitize and collect the attribute information for disaster mapping and response, many data collectors moves town to town to collect the attributes information. The attribute enrich satellite images and arieal photography will ease and speedup their data collection needs.

- Panchromatic band is lesser use as compare to the multispectral bands. This technique will make the panchromatic band in greater use. The value and price of panchromatic (PAN) band will level up the multispectral bands.

- Intelligent satellite images cost and demand will raise.

- Such images will act as a primary data source for all GIS platforms, companies who deal with GIS application will acquire such images at 1st instance for attribute data.

- Data integrity and accuracy will increase, accuracy of attribute data collected from such images is in fact, accuracy of satellite image.

- Space born mission and rescue teams will benefit from physical labels. Monitoring the cities through air is another benefit of this effort.

- This will lead to new image processing algorithms which can be use to extract attribute information within minutes.

\subsection{Disadvantages}

The following limitations and troubles are expected of this project.

- This project does not span on days or months for its complete implementation, we need years to implement it. so, it's not a quick fix.

- Implementing it at world scale demands a very organized and prolong advertisement and awareness. We need to make people aware of it.

- Standards are needed to be defined for physical labeling schemes, otherwise whole project can be a mess up.

\section{Promotion and Advertisement}

This is a very hard and sensitive part of this project. How to implement and advertise this project at large scale? And who is responsible to do this job? Again question is hard to answer.

But I am very much inclined if the following steps have to be taken this thing is not beyond its implementation.

- At country level, the national administration can initiate this project at 1st priority. Even national govt. can launch it at state level, as a state responsibility to standardized, manage, advertise and promote the cause as a national cause.

- Govt. And commercial agencies running high resolution space sensor to standardize, advertise and initiate the project.

- In developing countries, geospatial companies and disaster mapping agencies can initiate the project with help of local administration.

- Advertisement can be spark and spread through print media, electronic media, social media etc...

- Major GIS platforms like ESRI and open source can also contribute through online training and seminars.

- Initiate this project from the cities which are very much prone to natural disaster like cities under threat of tsunami, Catherina, earthquake and flooding.

- "Start from home". Other will follow it as a fashion and need. If once initiated it will spread like an epidemics, 
people and companies will catch it like flu.

\section{Advertisement Thoughts}

The following advertisement conclusion is part of mind; I do consider even some best mind can do it far batter then mine.

- One label for space segment please

- Let the satellite map you

- Advertise spatially advertise globally

- Let satellite know you

- Get batter rescued via satellite

- Do you have label on your roof?

\section{Conclusions}

This work is very much inclined and agrees with all those data collection modern techniques, which are part of system now. This is something new; again I would say it is a very hard job to implement, but its fruit will be beyond the imaginations if once started. In the next five years, if we became able to fully implement it, it will give us many advance GIS and remote sensing applications. Data collection would be an easier job through high resolution satellite imagery. One fear may raise that it may replace the old method and techniques, even some newer and more advanced systems may consider it a threat to their existence, but I am very much satisfied that dynamic features which change on daily basis would not let the other methods die young.

Expert opinions, suggestions and even criticism are more welcome.

\section{References}

[1] Xia, G.-S., Yang, W., Delon, J., Gousseau, Y., Sun, H. and Maître, H. (2010) Structural High-Resolution Satellite Image Indexing. ISPRS TC VII Symposium-100 Years ISPRS, Vienna.

[2] http://www.beachmarketing.co.uk/portfolio posts/northampton-borough-council

[3] http://www.ask.com/question/how-much-are-original-sindy-dolls-from1960s-with-sindy-ingrained-on-head

[4] http://www.mycasaspain.co.uk/restaurants.html

[5] http://ibnlive.in.com/news/fastfood-logos-light-up-childrens-brains/295434-17.html

[6] http://www.freewebs.com/robbo_05/armycadetforce.htm

[7] http://ww1.prweb.com/prfiles/2008/10/09/1452864/0_GeoEye-1_50cm_Kutztown_Univ_07-OCT-2008_Rev1.jpg

[8] http://apollomapping.com/blog/monthly-update-on-astriums-high-resolution-satellite-pleiades-1a-2

[9] Geoeye-1 Google Earth London UK.

[10] Bing Satellite Imagery, Sydney Australia. http://en.wikipedia.org/wiki/Hoyts

[11] Google Earth Street Views Sydney Australia. Google Earth Satellite Imagery Sydney Australia.

[12] http://www.dhi-gras.com/Products/SatelliteImages.aspx 